\title{
Desarrollo del dobles de juniors: Un enfoque progresivo en cancha basado en hechos y estadísticas
}

\author{
Michelangelo Dell'Edera, Luigi Bertino y Donato Campagnoli
}

\section{RESUMEN}

El dobles de juniors es una disciplina no muy desarrollada, ni muy considerada en los programas de entrenamiento de tenis. La Federación Italiana de Tenis llevó a cabo un estudio para evaluar ciertos parámetros del dobles a nivel junior, y compararlos con los del tenis profesional.
Palabras clave: : junior, dobles, cancha, tenis adaptado

Recibido: 2 Mayo 2018

Aceptado: 5 Junio 2018

Autor correspondiente:

Michelangelo Dell'Edera.

Correo electrónico:

m.delledera@libero.it

\section{INTRODUCCIÓN}

Se compilaron algunos datos estadísticos por medio del análisis de los partidos de dobles entre jugadores menores de 14 años (niños y niñas) en los campos nacionales de entrenamiento realizados regularmente por la Federación Italiana de Tenis, en el Centro Nacional de Tenis de Tirrenia, Italia (Centro di Preparazione Olimpica di Tirrenia). Los partidos analizados se jugaron tanto en cancha de tamaño "normal" $(23,77 \times 10,97 \mathrm{~m})$ como en cancha de "individuales" (23,77 x $8,23 \mathrm{~m})$.

El análisis de estos datos demostró que en la cancha "normal" los jugadores menores de 14 no desarrollan las situaciones ofensivas $y$, en consecuencia, tampoco las habilidades ofensivas ya que estas condiciones suelen no recompensar a los jugadores que van a la red, que practican un juego de transición o soluciones ganadoras, pero sí promueven soluciones tácticas/ estratégicas más defensivas y conservadoras.

\section{RESULTADOS}

Comencemos analizando los diferentes parámetros tácticos.

En la Figura 1 vemos, sorprendentemente, que el porcentaje de $1^{\circ}$ servicios en dobles de juniors en cancha "normal" es muy superior (59\%) al medido en la cancha de "individuales" (55\%) y el mismo que en dobles de la ATP (54\%).
En realidad, esto no debería sorprender, por el contrario, y necesariamente lleva a las siguientes conclusiones: en el dobles de juniors el enfoque táctico de quien sirve suele ser conservador, por lo cual, el nivel de riesgo en el primer servicio es bajo. Reducir en cambio, el ancho de la cancha estimula la actitud proactiva del servidor para el primer servicio, y los datos obtenidos se acercan a los de los jugadores profesionales.
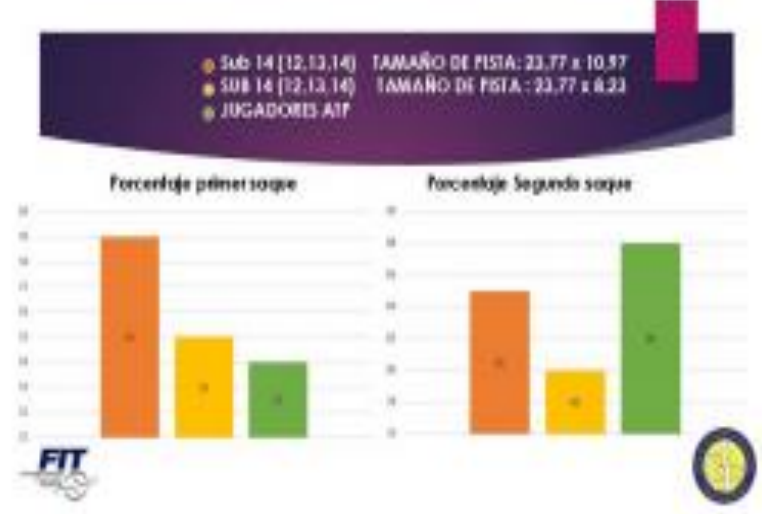

Figura 1. Porcentaje de primeros servicios y porcentaje de segundos servicios.

Un porcentaje menor de segundos servicios (80\%) en cancha de "individuales" probablemente indique que en este caso, también, el intento de asumir un riesgo se incrementa con respecto a la cancha "normal" (85\%)

La Figura 2 muestra en el gráfico de la derecha el porcentaje de servicios ganadores. El valor medido en la cancha "normal" (6\%) es mucho menor que el de la cancha de "individuales" 
(12\%). No obstante, los datos de los jugadores profesionales se mantienen muy alejados, con $28 \%$, debido al mayor desarrollo físico de los jugadores de este nivel.

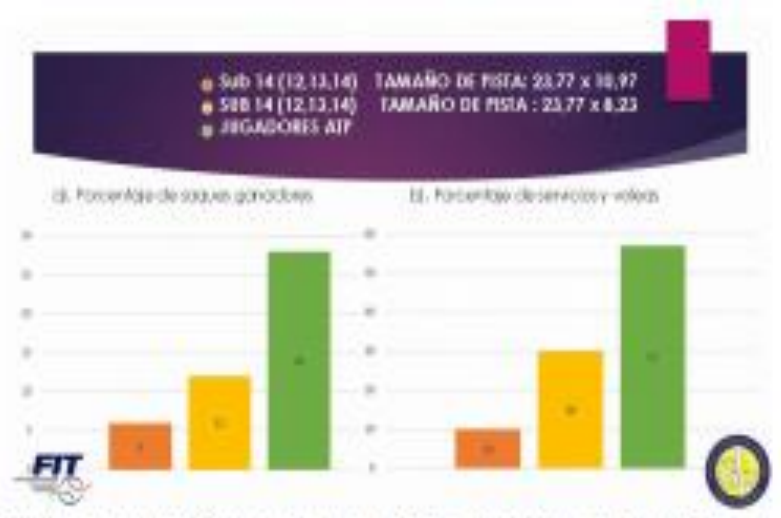

Figura za y $2 b$. Porcentaje de servicios ganodares y porcentaje de servicios y voleas.

El porcentaje de "servicio y volea" aún aumenta de 10 a 30\%, tendiendo hacia los valores de los jugadores profesionales (57\%).

Tanto los primeros como los segundos datos de la Figura 2 muestran que la tendencia a la búsqueda de soluciones ofensivas $y$ definitivas con el servicio aumenta considerablemente si se disminuye el ancho de la cancha.

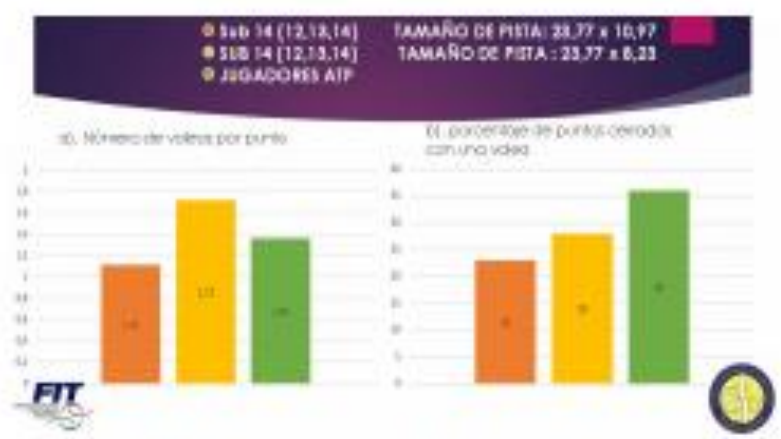

Figura $3 a$ y $3 b$. Nümero de voleas por punto y porcentaje de puntos cerrados con una voled.

Con el mismo tamaño de la cancha, aún los puntos que terminan con una volea ganadora incrementan del $23 \%$ al $28 \%$.

Los datos estadísticos de la Figura 3 confirman las tendencias anteriores: las elecciones tácticas y los patrones de juego se tornan más ofensivos, se acercan a los de los profesionales cuando se juega el dobles en una cancha de ancho reducido.

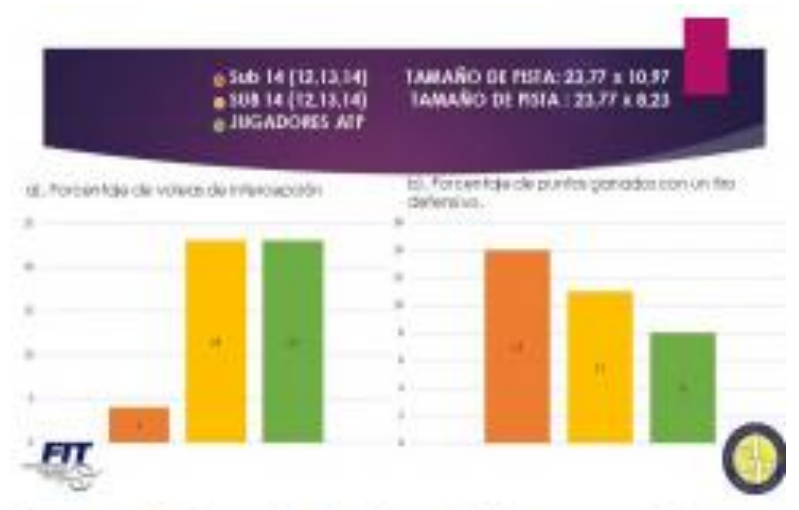

Figura sa y 4 b. Porcentaje de voleas cruxindose y porcentaje de punto: ganadas con un tion defensivo.

Los datos mostrados en la Figura 4 (gráfico de la izquierda) destacan el juego de red.

En la cancha de "individuales" el porcentaje se eleva a $23 \%$, igual que en los profesionales. Cuando se juega dobles en la cancha "normal", el juego de red es solamente el 6\%. En el gráfico de la derecha vemos que el porcentaje de puntos ganados con una acción táctica defensiva disminuye (de 14\% a $11 \%)$, y esto es como consecuencia de la actitud más agresiva de los jugadores en la cancha sin callejones.

\section{CONCLUSIONES}

Nuestra proposición de enseñanza consiste en cambiar el tamaño de la cancha, quitando los pasillos, y modificar la posición del servidor, haciendo que ejecute el servicio dentro de la cancha.

Estas dos modificaciones harán que los niños/niñas ejecuten naturalmente patrones tácticos de ataque como "Saque y Volea", volea, "volea robada" ("poach volley") y todos los patrones de ataque en general.

Los entrenadores de tenis propondrán ejercicios de dobles utilizando la cancha de "individuales", y quien sirve lo hará desde una posición avanzada (1-2 $\mathrm{m}$ dentro de la línea de fondo).

Nos referimos, entonces, a ejercicios con "pelota muerta" (incluyendo ejercicios desde el cesto), "ejercicios con peloteo" y ejercicios simulando "juego de partidos", ejercicios todos en los cuales los jóvenes jugadores puedan desarrollar gradualmente todas las habilidades requeridas para jugar un dobles de alto nivel, por medio de progresiones técnicotácticas específicas.

CONTENIDO ITF ACADEMY RECOMENDADO (HAZ CLICK ABAJO) 
Derechos de Autor (c) 2018 Michelangelo Dell'Edera, Luigi Bertino y Donato

$$
\text { Campagnoli }
$$

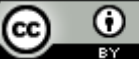

Este texto está protegido por una licencia CreativeCommons 4.0 .
Usted es libre para Compartir —copiar y redistribuir el material en cualquier medio o formato- y Adaptar el documento — remezclar, transformar y crear a partir del material- para cualquier propósito, , incluso para fines comerciales, siempre que cumpla la condición de:

Atribución: Usted debe dar crédito a la obra original de manera adecuada, proporcionar un enlace a la licencia, e indicar si se han realizado cambios. Puede hacerlo en cualquier forma razonable, pero no de forma tal que sugiera que tiene el apoyo del licenciante o lo recibe por el uso que hace de la obra.

Resumendelicencia - Textocompletodelalicencia 